\title{
Muon-induced neutron production and detection with GEANT4 and FLUKA
}

\author{
H. M. Araújo ${ }^{a, *}$, V. A. Kudryavtsev ${ }^{\text {b }}$, N. J. C. Spooner ${ }^{b}$, \\ T. J. Sumner ${ }^{a}$

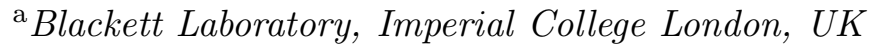 \\ ${ }^{\mathrm{b}}$ Department of Physics $\&$ Astronomy, University of Sheffield, UK
}

\begin{abstract}
We report on a comparison study of the Monte Carlo packages GEANT4 and FLUKA for simulating neutron production by muons penetrating deep underground. GEANT4 is found to generate fewer neutrons at muon energies above $\sim 100 \mathrm{GeV}$, by at most a factor of 2 in some materials, which we attribute mainly to lower neutron production in hadronic cascades. As a practical case study, the muon-induced neutron background expected in a $250 \mathrm{~kg}$ liquid-xenon WIMP dark matter detector was calculated and good agreement was found for the recoil event rates. The detailed model of neutron elastic scattering in GEANT4 was also shown to influence the nuclear recoil spectrum observed in the target, which is presently a shortcoming of FLUKA. We conclude that both packages are suited for this type of simulation, although further improvements are desirable in both cases.
\end{abstract}

Key words: GEANT4, FLUKA, Monte Carlo simulations, dark matter, muon interactions, neutron production PACS: 21.60.Ka, 24.10.Lx, 14.60.Ef, 14.20.Dh, 14.80.Ly, 13.60.Rj, 25.20.-x, 25.30.c, 25.40, 98.70.Vc

\section{Introduction}

The knowledge of neutron fluxes in underground laboratories is crucial in experiments searching for and detecting rare events associated with neutrino interactions, double-beta decay and dark matter WIMPs (Weakly Interacting

\footnotetext{
* Corresponding author; address: Astrophysics Group, Blackett Laboratory, Imperial College London, SW7 2BW, UK

Email address: H.Araujo@imperial.ac.uk (H. M. Araújo).
} 
Massive Particles). For example, in dark matter searches, in order to reach $10^{-10} \mathrm{pb}$ sensitivity to the WIMP-nucleon cross-section, the neutron flux produced by muons underground must be known and suitably attenuated. Other sources of neutrons can, in principle, be mitigated by using radio-pure materials and components together with appropriate shielding. In many existing underground laboratories (Gran Sasso, WIPP, Kamioka, Modane, Boulby and others) the muon-induced neutron flux may be a key limiting factor for detector sensitivity and may require an active veto system to be installed around the target. This can easily be the dominant cost in a large detector system.

Atmospheric muons, generated in Extensive Air Showers at high altitudes in the atmosphere, penetrate deep underground and produce neutrons in the rock surrounding a laboratory or in the materials inside it. Most neutrons do not come directly from muon-nucleus spallation reactions, but from the muoninitiated hadronic and electromagnetic cascades. These neutrons spread away from the primary muon track, thermalising slowly, and finally get captured by protons or other nuclei. Some, however, can strike a nucleus in the target volume transferring sufficient energy to be detected. This is an irreducible background for many underground experiments. In dark matter searches, for example, the single nuclear-recoil signals from neutron elastic scattering cannot be distinguished from WIMP interactions. An increased gamma activity of some materials is also expected from neutron activation.

Underground neutron fluxes are difficult to measure since they are overwhelmed by gamma-rays from local radioactivity. In addition, most neutrons at the aforementioned underground laboratories come from rock radioactivity: muoninduced neutrons contribute typically less than one percent to the total, unshielded neutron flux. Although these neutron events may have a clear signature of an associated muon and/or cascade, large detectors are needed to study them with sufficient accuracy, especially to understand the neutron behaviour at large distances from the muon track. Monte Carlo (MC) simulations are thus crucial in designing high-sensitivity detectors. Naturally, this reliance on simulation alone must be accompanied by validation of the codes against other experimental data, and by comparison between different packages.

FLUKA [1] is a well-established simulation tool in nuclear and particle physics, and has been used for neutron calculations in a variety of experiments. However, it does not treat the elastic scattering of neutrons at low energies with sufficient detail (on an event-by-event basis) for the purpose of dark matter experiments. So-called 'Kerma' factors are used to generate energy deposition from nuclear recoils (other than protons), which are equivalent to the average recoil energy for a certain neutron energy. The GEANT4 [2] toolkit can potentially be used for end-to-end simulations of experiments, from background calculations down to detailed detector characterisation. Its object-oriented design and open-source nature make it rather flexible. Critically, it generates and 
tracks in a realistic way the recoiling nuclei from individual neutron elastic interactions.

In this paper we compare GEANT4 and FLUKA simulations of neutron production, and validate them against available experimental data. Some FLUKA results have already been published in Ref. [3] (hereafter 'Paper 1') and Ref. [4] (Paper 2). We believe this to be a useful test of GEANT4 physics for lowbackground experiments, an area where it finds increasing application. We describe the full simulation of the neutron background in an idealised WIMP dark matter detector, hypothetically located at the Boulby mine (2.8 km w.e.) - location of the UK Dark Matter Collaboration experiments. In particular, we compare the underground neutron spectrum, veto and shielding efficiencies and nuclear recoil spectrum expected in a $250 \mathrm{~kg}$ xenon target with the FLUKA results published in Ref. [5] (Paper 3). Muon propagation from the surface is not addressed here. Instead, the MC code MUSUN [3] was used to calculate the muon energy spectrum and angular distribution at Boulby in the way described in Paper 3.

The present simulations made use of GEANT4 release 6.2 and FLUKA-2003. The FLUKA modelling described in Paper 3 was performed with FLUKA2002, whereas the results of Paper 1 and Paper 2 were obtained using FLUKA1999.

\section{Muon-induced neutron production}

Since GEANT4 offers alternative models to treat certain physics processes, we mention here briefly which ones were used in these simulations. The physics implemented in the toolkit is fully described in Ref. [6]. Muon-induced spallation (or muon photonuclear interaction) is modelled above $1 \mathrm{GeV}$ muon energy; the final-state generator relies on parameterised hadronic models. Gamma inelastic scattering, the real photonuclear interaction, generates its hadronic final states using a chiral-invariant phase-space decay model below $3 \mathrm{GeV}$; a theoretical quark-gluon string model simulates the punch-through reaction at higher energies. The hadronic interaction of nucleons, pions and kaons is simulated with the quark-gluon string model above $6 \mathrm{GeV}$, an intra-nuclear binary cascade model at lower energies and a pre-equilibrium de-excitation stage below $70 \mathrm{MeV}$. The equilibrium stage considers fragment and gamma evaporation, fission, Fermi break-up and multi-fragmentation of highly-excited nuclei. Neutron transport and interactions are described by data-driven models below $19 \mathrm{MeV}$.

The choice of electromagnetic physics is more straightforward and will not be described here, except to say that the so-called 'Low Energy' package was 
used for $e^{-}$and gammas interactions, and the standard models were applied to the remaining particles (including muons). The production thresholds ('cuts') considered in these simulations were a few tens of $\mathrm{keV}$ for gammas and $\sim 1 \mathrm{MeV}$ for $e^{-}$and $e^{+}$in all materials. No thresholds were applied to neutron tracking.

\subsection{Neutron production in hydrocarbon scintillator}

In order to study neutron production by muons in several materials we consider a $\mu^{-}$beam incident at the centre of a block with thickness $3200 \mathrm{~g} / \mathrm{cm}^{2}$ and comparable transverse dimensions. We compared the muon energy spectra at the end of the block from GEANT4, FLUKA and the muon propagation code MUSIC [7] and found them to be in good agreement [8]. This validates the methods used to calculate muon interaction cross-sections.

Muon-induced cascades require a certain length of material to develop and reach equilibrium in the number of neutrons produced per unit muon track length. To avoid this and other edge effects, only neutrons produced in the central half-length of the block are considered. In counting the number of neutrons created in a cascade one must avoid double-counting inelastically scattered ones, which often share the final state with newly generated neutrons. In the GEANT4 simulation we consider that the highest energy one in the final state corresponds to the initial neutron ${ }^{1}$ and remove it from the count (akin to the concept of 'stars' in FLUKA). For muon energies below $\sim 100 \mathrm{GeV}$ one must also correct for muon energy loss, since the neutron yield decreases noticeably as the muon progresses through the material. These cases are corrected by extrapolating the trend of the decreasing yield to the beginning of the muon beam.

A generic hydrocarbon with composition $\mathrm{C}_{n} \mathrm{H}_{2 n}$ (density $\rho=0.8 \mathrm{~g} / \mathrm{cm}^{3}$ ) was the lightest material simulated in this study. This can represent commonly used neutron-shielding hydrocarbons as well as typical organic scintillators (e.g. $\mathrm{C}_{10} \mathrm{H}_{22}$ ) for which experimental data are available. The total neutron yields, which include neutrons of any energy, are shown in Fig. 1 as a function of muon energy (the MC statistical uncertainties are comparable to the size of the data markers). Although GEANT4 results agree, at higher energies, with the power law $E^{0.74-0.79}$ predicted by FLUKA in Papers 1 and 2, there is an enhancement with decreasing energy relative to the previous FLUKA simulations. Present results with FLUKA-2003 show similar enhancement of neutron production at low energies over the simple power law. Both GEANT4

1 Strictly, the final state of the inelastic interaction has no memory as to which neutron corresponds to the incident one, and so this assertion does not always hold. We note, however, that this will not affect the neutron multiplicity (and hence the total yield), only the production spectrum. 


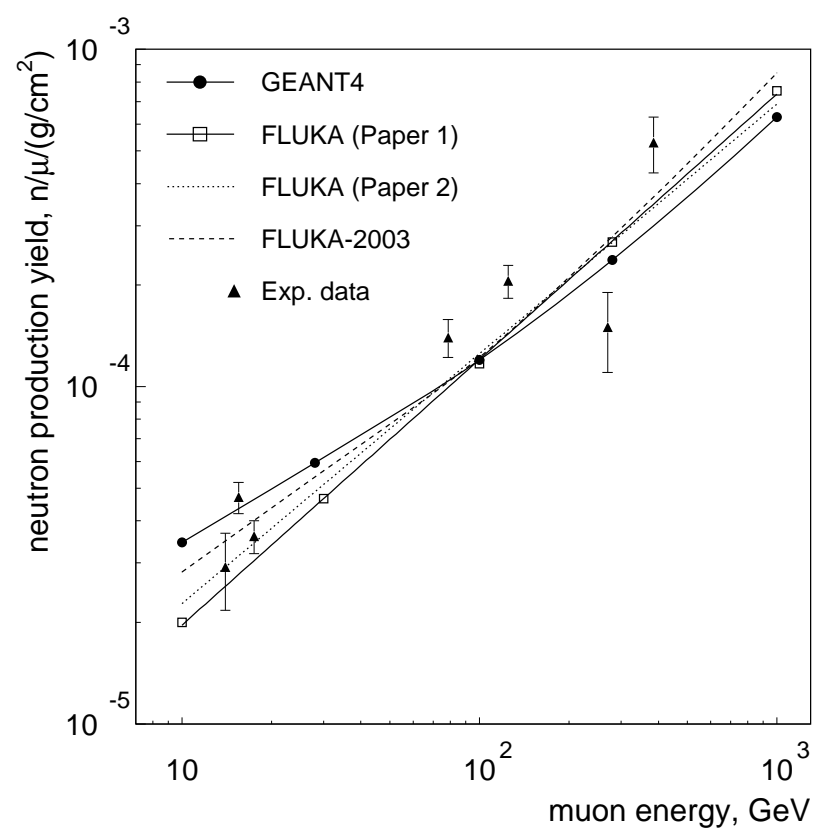

Fig. 1. Dependence of the neutron yield per unit muon track length on muon energy for $\mathrm{C}_{10} \mathrm{H}_{20}$ scintillator. The experimental data represent measurements at depths varying between $20 \mathrm{~m}$ w.e. and $5200 \mathrm{~m}$ w.e. with the corresponding underground muon spectrum. Here they are plotted as a function of the mean muon energy. This approximation is justified in Paper 1 and Paper 2, where references to the original experiments can be found. Note the higher neutron production rate at low energies in present (FLUKA-2003) simulations compared to Paper 1 and Paper 2.

and FLUKA are consistent with the experimental data shown, which have been measured at various depths around the world, keeping in mind the spread in experimental results.

We have also analysed the importance of individual neutron-producing processes in this simulation, which can be compared to similar data for FLUKA presented in Papers 1 and 2. The relative contributions of the most important mechanisms to the total neutron rate are shown in Fig. 2 for GEANT4. There is a degree of agreement between the two codes, in that both predict neutron production in electromagnetic cascades (real photonuclear interaction) to dominate at lower energies and to decrease in importance with increasing muon energy, and generation in hadronic cascades to become more important with increasing muon energy. Both codes confirm that most neutrons are not produced in direct muon-induced spallation, but rather in the cascades muons initiate, and more so at higher energies. However, the GEANT4 results reveal a greater dominance of electromagnetic cascades at low energies (higher total neutron yield, cf. Fig. 1), and this scenario is not significantly different at high energies, where FLUKA predicts neutron production in hadronic cascades to take over. In this material, although GEANT4 appears to overproduce neutrons in electromagnetic cascades, it underproduces in hadronic 


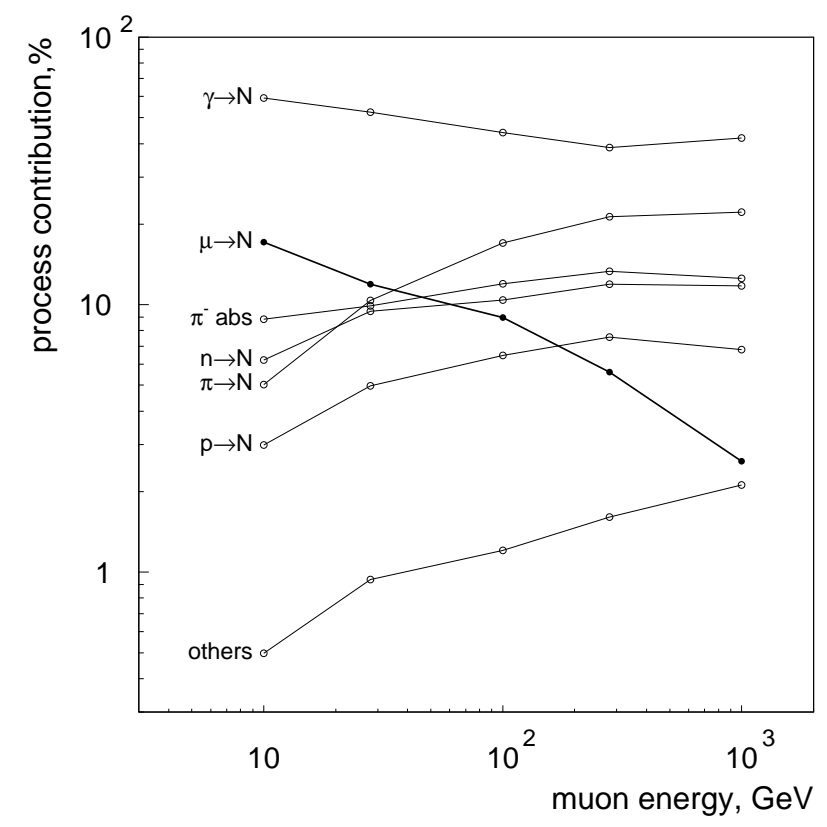

Fig. 2. Relative contribution of individual processes to the total neutron yield in scintillator from the GEANT4 simulation. The processes shown explicitly are photonuclear interaction of gammas $(\gamma \rightarrow N)$, muon spallation $(\mu \rightarrow N)$, proton spallation $(p \rightarrow N)$, pion $\left(\pi^{+}\right.$and $\left.\pi^{-}\right)$spallation $(\pi \rightarrow N), \pi^{-}$absorption at rest $\left(\pi^{-}\right.$abs $)$and neutron inelastic scattering $(n \rightarrow N)$. The processes gathered under 'others' include electronuclear reactions $\left(e^{ \pm} \rightarrow N\right)$, kaon spallation and $\mathrm{K}^{-}$absorption at rest, as well as spallation reactions involving light fragments $\left({ }^{2} \mathrm{H},{ }^{3} \mathrm{H},{ }^{3} \mathrm{He}\right.$ and $\alpha$-particles $)$, anti-nucleons $(\bar{n}, \bar{p})$ and short-lived hadrons $(\Lambda, \Sigma, \Xi$, etc.).

cascades compared to FLUKA, giving similar total yield in both codes. It should be noted that this could be due to a difference in the total interaction cross-sections, in the neutron-production cross-sections or in the final-state multiplicity of secondary particles.

The neutron energy spectrum at production in the scintillator, calculated in the way described above for the total neutron yield, is shown in Fig. 3 for $280 \mathrm{GeV}$ muons, a value close to the average muon energy at several underground labs. These data are compared with a parameterisation of the relative spectrum given in Paper 2 for FLUKA. Measurements with the LVD detector [9], located at the Gran Sasso underground laboratory (mean depth of $3.6 \mathrm{~km}$ w.e.), are also shown — normalised for visual agreement. Although these experimental data do not show the neutron energy spectrum at production, but a volume-averaged spectrum instead, they are nevertheless a useful guide. Note that the parameterisation of the neutron spectrum given in Paper 2 does not show a feature above $\sim 10 \mathrm{MeV}$ due to the Giant Dipole Resonance (GDR) and the so-called 'Quasi-Deuteron' region. This feature is, however, also seen in the FLUKA spectrum, averaged over the entire scintillator volume, presented in Paper 1. 


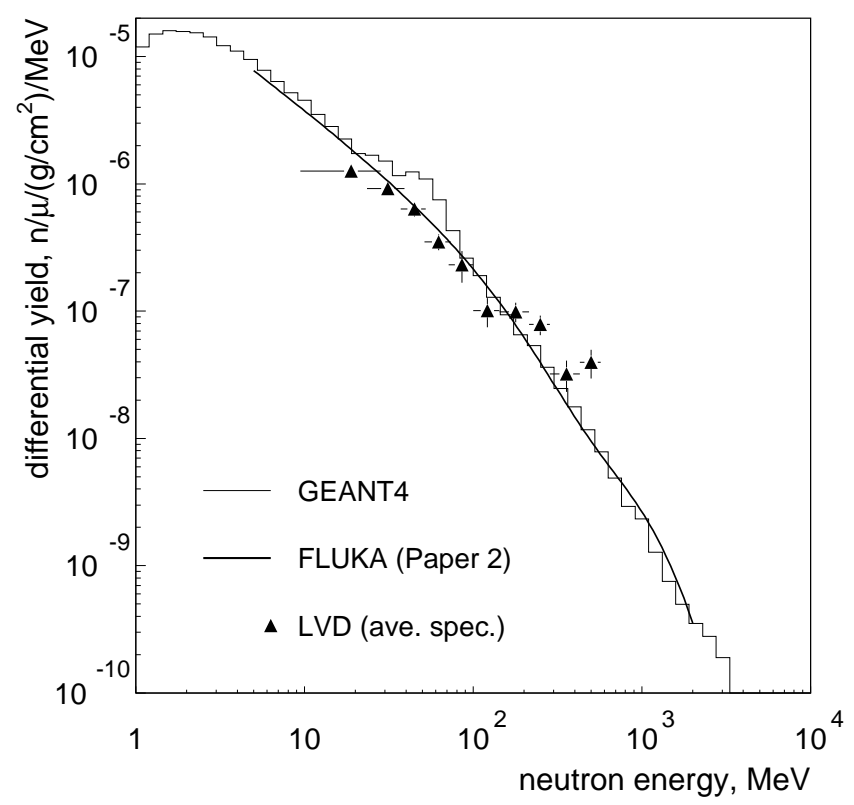

Fig. 3. Differential energy spectrum of muon-induced neutrons produced in scintillator. The GEANT4 absolute yield is compared with the relative FLUKA spectrum represented by the parameterisation given in Paper 2. Experimental data refer to an average neutron spectrum in the scintillator volume as measured by the LVD experiment [9]. The FLUKA and LVD datasets were normalised to the GEANT4 spectrum for visual agreement.

Neutrons can be created some distance away from the primary muon track, an issue to consider when modelling veto performances. The FLUKA 'star density' output is an estimator for the spatial distribution of neutron reactions, which can be compared to experimental measurements carried out with large scintillator volumes - as reported in Paper 1. A FLUKA 'star' is a hadronic inelastic interaction (neutron spallation, in this instance) above a certain energy threshold. An equivalent quantity was calculated from the GEANT4 dataset, which was obtained for the input muon spectrum expected at $2.8 \mathrm{~km} \mathrm{w.e.} \mathrm{un-}$ derground. The result is shown in Fig. 4. Both simulations agree well with the LVD measurement [9]. More recent LVD data [15] suggest a higher number of neutrons at large distances from the muon track. Note, however, that LVD is not a detector with a single uniform medium but has a modular structure with gaps between modules. Thus any such comparison should be considered with caution.

\subsection{Neutron yields in other materials}

Neutron production in several other materials of interest to low background experiments was studied with GEANT4 for a fixed muon energy of $280 \mathrm{GeV}$, 


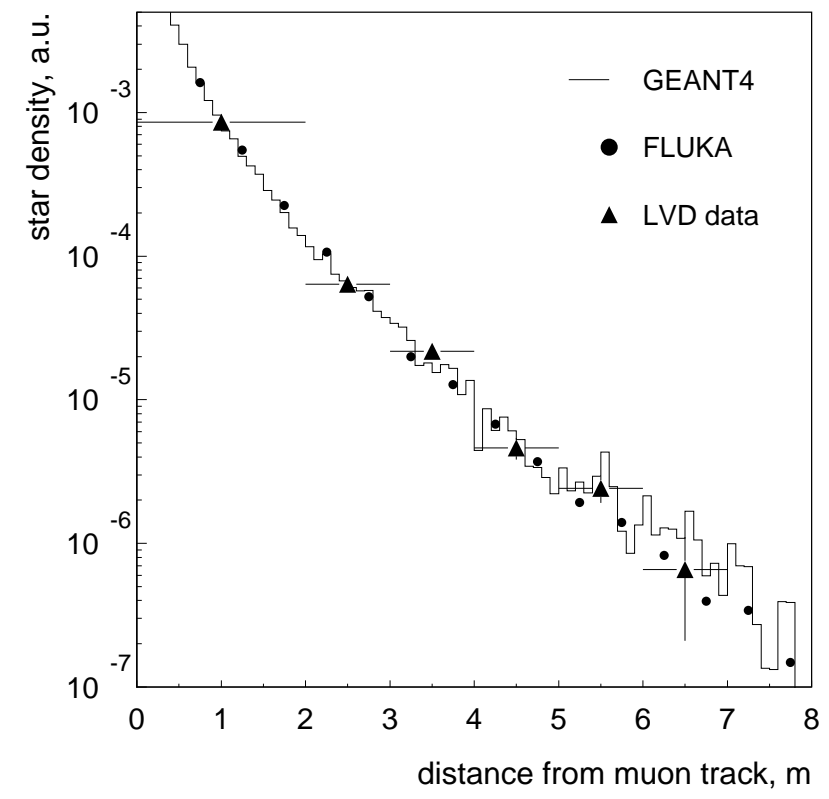

Fig. 4. Lateral distribution of neutron inelastic interactions from the primary muon track for the muon spectrum at about $2.8 \mathrm{~km}$ w. e. (280 GeV mean energy). The FLUKA 'star density' is compared with LVD data [9] and with a similar quantity calculated with GEANT4. Normalisation is done to the GEANT4 distribution for visual agreement at small distances.

using the block geometry and the neutron event selection rules described previously for organic scintillator. The variation of the total neutron yield with atomic weight of the element (or average atomic weight for compounds) is shown in Fig. 5, along with the FLUKA results obtained in Paper 1 (we also show new results for scintillator and lead from FLUKA-2003 simulations). The two codes differ at most by a factor of 2 - with FLUKA predicting consistently higher rates at this energy — but the largest discrepancies occur precisely for materials of great interest for underground experiments: notably $\mathrm{NaCl}$ (the rock at WIPP and Boulby), Fe and Pb (shielding materials). The statistical uncertainty associated with each data point is much smaller than the discrepancy between corresponding FLUKA and GEANT4 data points. The rates can be fitted by a power-law dependence of the atomic weight $A$ : $R=b A^{\beta}$. We obtain $b=(3.0 \pm 0.4) \times 10^{-5}$ and $\beta=0.82 \pm 0.03$ for the GEANT4 simulation, while Paper 1 reports $b=(5.33 \pm 0.17) \times 10^{-5}$ and $\beta=0.76 \pm 0.01$ for FLUKA.

Valuable information can be gained by decomposing the total neutron rates into the originating physical processes and studying how these vary with atomic weight. The data in Fig. 6 break down the contribution of five inelastic scattering processes at $280 \mathrm{GeV}$ muon energy. Real photonuclear interactions clearly dominate in all materials, increasing linearly with $A$. This is indeed the dependence expected for the total cross-section, suggesting that the number of 


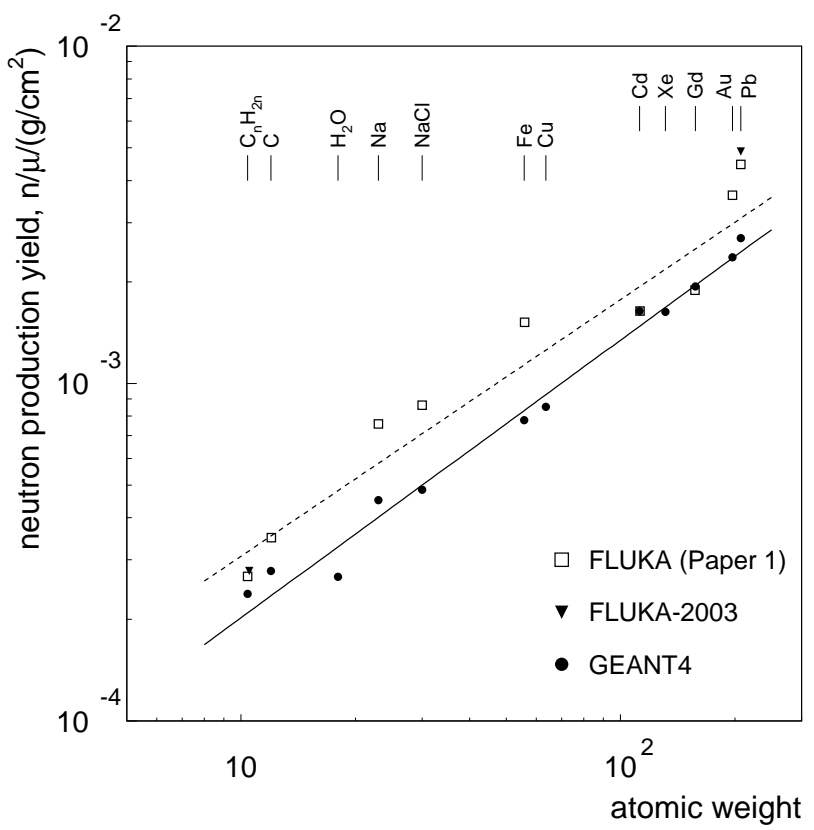

Fig. 5. Dependence of the neutron yield on the average atomic weight of the material in FLUKA (Paper 1) and GEANT4 (this work) for $280 \mathrm{GeV}$ muons, for the elements and compounds indicated. The power-law parameterisations are given in the text. Two data-points show the present FLUKA-2003 results for scintillator and lead.

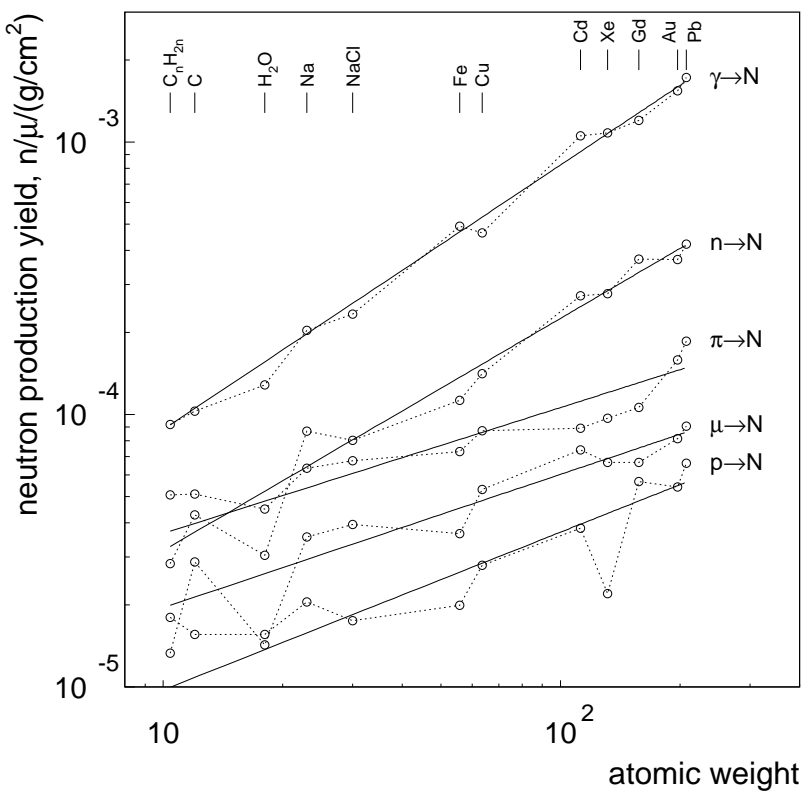

Fig. 6. Contribution of different processes to the neutron yield as a function of atomic weight of the material for the GEANT4 simulation of $280 \mathrm{GeV} \mu^{-}$. The processes include photoproduction $(\gamma \rightarrow N)$, neutron inelastic scattering $(n \rightarrow N)$, $\pi^{+}$and $\pi^{-}$spallation $(\pi \rightarrow N)$, muon spallation $(\mu \rightarrow N)$ and proton spallation $(p \rightarrow N)$. 
neutrons produced per reaction remains approximately constant — confirmed by the GEANT4 team [16]. Photoproduction in FLUKA gives less neutrons in scintillator $\left(6.5 \times 10^{-5}\right.$ compared to $9.2 \times 10^{-5} \mathrm{n} / \mu /\left(\mathrm{g} / \mathrm{cm}^{2}\right)$ in GEANT4 $)$ and more neutrons in lead $\left(2.4 \times 10^{-3}\right.$ and $1.7 \times 10^{-3} \mathrm{n} / \mu /\left(\mathrm{g} / \mathrm{cm}^{2}\right)$, respectively). Secondary neutron production in GEANT4, resulting from neutron inelastic scattering, increases slightly more slowly $\left(A^{0.85}\right)$ than photoproduction. Yields from pion $\left(\pi^{+}, \pi^{-}\right)$, proton and muon spallations vary approximately as $A^{0.5}$. The scattered nature of the data for a given process is not statistical in origin. The relative error for direct muon spallation (which also governs hadronic cascade statistics), is only a few percent.

In conclusion, muon-induced neutron production in GEANT4 is generally lower than that in FLUKA at high muon energies, although this difference is typically smaller than a factor of 2 - an acceptable agreement for many purposes. This is due to a generalised neutron deficit from hadronic cascades. This underproduction with respect to FLUKA may be expected, since the latter does not model the emission of fast nuclear fragments from highly-excited nuclei (multi-fragmentation), especially important in heavy nuclei, thus leaving more energy available for neutron evaporation [16]. In heavy elements electromagnetic cascades also underproduce neutrons with respect to FLUKA but this is not the case for the light hydrocarbon scintillator. Direct production in muon-nucleus interactions is never dominant. Nevertheless, we analyse this process more closely in the following section.

Experimental data for neutron production by energetic muons in heavy targets is scarce and not straightforward to interpret without detailed modelling, given the complicated experimental set-ups and poor knowledge of the neutron detection efficiencies. In Ref. [17] FLUKA simulations of neutron production by high-energy muons were compared to experimental measurements in lead $[18,19]$. Some of these results relevant to high muon energies, converted to the neutron yield per muon are: $1.75 \times 10^{-3} \mathrm{n} / \mu /\left(\mathrm{g} / \mathrm{cm}^{2}\right)$ for a mean muon energy of $\simeq 110 \mathrm{GeV}$ (depth $800 \mathrm{~m}$ w.e.) [18] and $1.16 \times 10^{-2} \mathrm{n} / \mu /\left(\mathrm{g} / \mathrm{cm}^{2}\right)$ for a mean muon energy of $\simeq 310 \mathrm{GeV}$ (depth $4300 \mathrm{~m}$ w.e.). We used the muon energy dependence of the neutron yield ( $\propto E^{0.79}$ in hydrocarbon [3]) to convert the quoted neutron yields to those at $280 \mathrm{GeV}$ and to compare them to our simulations. We obtained $\sim 3.7 \times 10^{-3} \mathrm{n} / \mu /\left(\mathrm{g} / \mathrm{cm}^{2}\right)$ from Ref. [18] and $\sim 1.1 \times 10^{-2} \mathrm{n} / \mu /\left(\mathrm{g} / \mathrm{cm}^{2}\right)$ from Ref. [19]. The first result is in good agreement with the MC predictions (especially FLUKA's) — assuming that the total neutron yield was measured in the experiments - whereas the second clearly indicates a higher yield than the simulations. Moreover, the quoted target thickness for these experiments is too small for the muon-induced cascades to develop fully, thus reducing the number of detected neutrons and making direct comparison difficult. In conclusion, some experiments may suggest that both FLUKA and GEANT4 underestimate neutron production in heavy targets, although more conclusive measurements are required. 


\subsection{Neutron production by muon-induced spallation}

The inelastic scattering of muons off nuclei can be explained in terms of the exchange of quasi-real virtual photons - hence it is also known as the muon photonuclear interaction. Presently, the GEANT4 treatment of the muonnucleus interaction uses the total cross-section formulae given in Ref. [11] and Ref. [12] and the parameterisation of the real photonuclear cross-section $(\gamma-N)$ from Ref. [13]. This treatment applies to muon energies above $1 \mathrm{GeV}$ and a minimum energy transfer of $200 \mathrm{MeV}$ [6]. The final-state hadronic vertex uses parameterised hadronic models. Although FLUKA follows an approach similar to that currently adopted in GEANT4, it is nevertheless useful to compare the two models, and these with experimental data.

The CERN NA55 has produced relevant data for such a comparison. In this experiment analysis was performed of the production of fast neutrons by $190 \mathrm{GeV}$ muons in graphite, copper and lead [10]. Given the aim in NA55 of studying neutron production in direct muon-nucleus interactions, small targets were used in order to minimise secondary reactions. Three neutron detectors were located at $45^{\circ}, 90^{\circ}$ and $135^{\circ}$ from the muon beam, for which an energy threshold (in neutron energy) of $\simeq 10 \mathrm{MeV}$ was indicated. Simulations were thus set-up with FLUKA and GEANT4 to model the three targets. The angular distribution of neutrons emitted from a target was recorded in order to calculate the differential cross-section for neutron production, which is shown in Fig. 7. GEANT4 results are given for two cases: firstly - data including only muon-induced spallation, and secondly - data from allowing the complete set of physics processes. The FLUKA simulation modelled only the latter case.

Several conclusions can be drawn from the data shown. Firstly, if either MC is to be believed, then the experiment could not measure direct muon-induced spallation exclusively. The contribution of secondary reactions cannot be neglected, especially for the carbon and copper targets. Secondly, both codes agree quite reasonably, in fact better than anticipated given the results presented in the previous section. Therefore the discrepancy observed for $\mathrm{Pb}$ in Fig. 5 does not arise from muon spallation - in fact, GEANT4 predicts a slightly higher fast-neutron yield than FLUKA for this element, as shown in Fig. 7 - but is instead associated with cascades (the main contribution in Fig. 5), which do not build up in thin targets. Finally, it is apparent that the experimental points lie far above the MC data. If the disagreement for the light materials is already unreasonable, the case of lead cannot be easily explained. In fact, even if a much lower neutron threshold of $1 \mathrm{MeV}$ is considered, both MC models would still predict a lower cross-section than those reported in Ref. [10].

The production of lower energy neutrons by deep inelastic scattering (DIS) of 


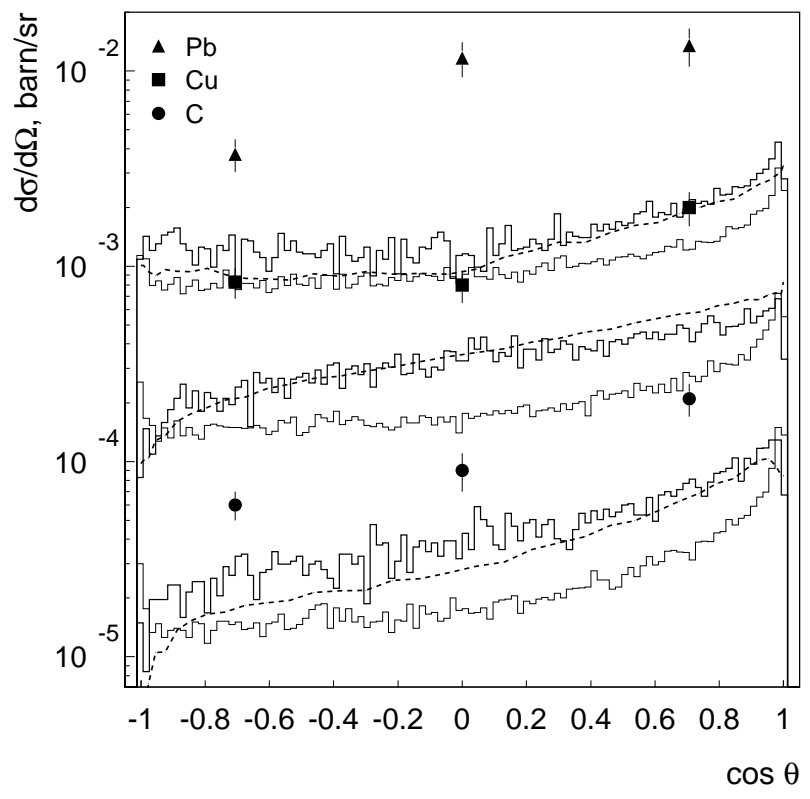

Fig. 7. Differential cross-section of neutron production by $190 \mathrm{GeV}$ muons for a $10 \mathrm{MeV}$ threshold in neutron energy. The data points represent the results of the NA55 experiment. The thin-line histogram shows the GEANT4 simulation considering muon-nucleus interaction only; the thick histogram includes all physics processes. The dashed line represents the FLUKA results for the latter case.

$470 \mathrm{GeV}$ muons in lead was studied by the E665 Collaboration, who found average neutron multiplicities per DIS event of $\simeq 5$ for neutron energies under $10 \mathrm{MeV}$ [20]. A value of 3.7 is obtained for the GEANT4 simulation of the $\mu-\mathrm{N}$ process at this muon energy, in reasonable agreement with the experimental result. The simulated neutron spectrum (per unit energy) exhibits a double-exponential behaviour below $10 \mathrm{MeV}$ - also in agreement with the experimental findings. The two decay constants, due to neutron evaporation from the thermalised nucleus and from pre-equilibrium emission, are characterised by nuclear temperatures of $0.93 \mathrm{MeV}$ and $3.7 \mathrm{MeV}$, compared to $0.7 \pm 0.05 \mathrm{MeV}$ and $5 \pm 1 \mathrm{MeV}$ obtained in E665. In conclusion, the spallation of neutrons under $10 \mathrm{MeV}$ as predicted by GEANT4 for lead does not conflict with these experimental data.

The role of the minimum energy transfer in the muon photonuclear models in neutron production was pointed out in Paper 2. This threshold comes about because the virtuality of the photon can no longer be neglected when it becomes comparable to its energy. Recently, the total $\mu-N$ cross-section was reported to increase by $2-3$ times if the minimum energy transfer is decreased from $140 \mathrm{MeV}$ to $10 \mathrm{MeV}$, based on the parameterisation used in FLUKA [21]. We have confirmed that this difference is only $10-15 \%$ greater for the $200 \mathrm{MeV}$ threshold in GEANT4. The aforementioned study also found that the parameterisation used to describe the $\gamma-N$ cross-section in FLUKA [12] (similar to 
that from Ref. [13] used in GEANT4) overestimates more rigorous theoretical calculations when extrapolated to low energy gammas. Consequently, the increase in the muon cross-section with decreasing threshold is not expected to be as large as mentioned above. In any case, as pointed out in Paper 2, we expect many more neutrons to be produced by bremsstrahlung (real) photons with low energies in electromagnetic cascades than by virtual ones in muon interactions with small energy transfers.

\section{Underground neutron fluxes: a case study}

The UK Dark Matter Collaboration (UKDMC) has been assessing the feasibility of a xenon-based tonne-scale dark matter experiment to be installed at the Boulby Underground Laboratory. In this context, initial calculations using FLUKA, reported in Paper 3, have so far been performed of the muon-induced background in a $250 \mathrm{~kg}$ xenon target. Building on that work we present here a case-study comparison between FLUKA and GEANT4. The calculated neutron fluxes and spectra at the rock/cavern boundary and after various shields are also relevant to other underground experiments in different laboratories.

\subsection{Energy spectrum at rock surface}

In these simulations an air-filled cavern (otherwise empty) with a height of $5 \mathrm{~m}$ and horizontal dimensions of $6 \times 6 \mathrm{~m}^{2}$ was located in a $20 \times 20 \times 20 \mathrm{~m}^{3}$ cube of pure $\mathrm{NaCl}\left(\rho=2.2 \mathrm{~g} / \mathrm{cm}^{3}\right)$, at a depth of $10 \mathrm{~m}$ from the top. NaCl represented the halite rock surrounding the laboratory. Muons were sampled on 5 sides of the salt cube, according to the energy spectrum and angular distribution calculated with the MUSUN Monte Carlo code for a rock overburden of $2800 \mathrm{~m}$ w.e. [3]. The muon flux experimentally measured underground, used to normalise the simulations, was $(4.09 \pm 0.15) \times 10^{-8} \mathrm{muons} / \mathrm{cm}^{2} / \mathrm{s}[22,23]$.

The GEANT4 and FLUKA results for the relative neutron rate (the number of neutrons per muon entering the cavern) are plotted in Fig. 8 for two cases: i) for neutrons crossing the interface for the first time and ii) also including those re-entering the laboratory after being scattered back from the rock. For the latter case, results agree within a factor of 2 for most of the energy range plotted, which is consistent with the level of agreement of the total yields found previously for $280 \mathrm{GeV}$ muons. In the range $1-10 \mathrm{MeV}$ - most interesting for a WIMP dark matter experiment - the agreement is even better. The integral neutron flux above $1 \mathrm{MeV}$ is $6.9 \times 10^{-10} \mathrm{n} / \mathrm{cm}^{2} / \mathrm{s}$ according to GEANT4, close to the FLUKA prediction of $8.7 \times 10^{-10} \mathrm{n} / \mathrm{cm}^{2} / \mathrm{s}$. 


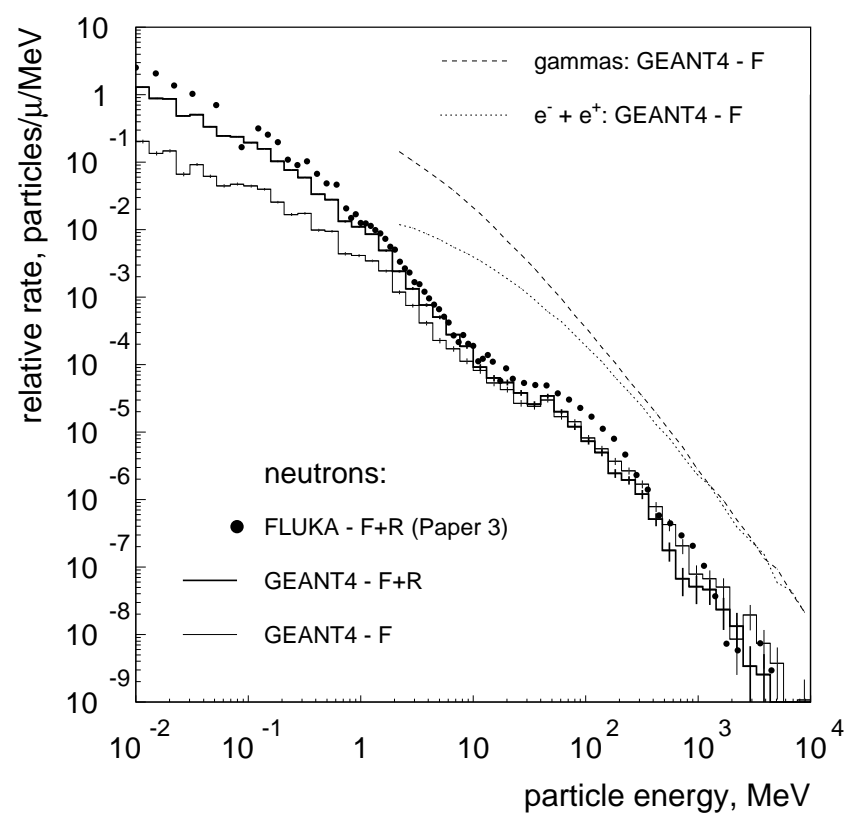

Fig. 8. Neutron energy spectrum at the rock/cavern boundary obtained with FLUKA and GEANT4. The curve labelled ' $\mathrm{F}$ ' includes only those neutrons entering the cavern for the first time; the curve marked ' $\mathrm{F}+\mathrm{R}$ ' accounts also for neutrons reflected back from the walls after crossing the empty laboratory. Also shown are the differential spectra for gammas and electrons $\left(e^{-}\right.$and $\left.e^{+}\right)$at the rock face.

The GEANT4 simulation also provided the gamma and electron/positron fluxes at the rock face, which are shown in Fig. 8. These data include all muon events, not just those producing neutrons in the cavern. Many more gammas than neutrons enter the experimental hall. In fact, most neutron events are accompanied by gammas (and $e^{ \pm}$) - assuming a minimum energy of $1 \mathrm{MeV}$ for both neutrons and gammas. Together with the primary muons, this electromagnetic component can help to tag neutrons emerging from the rock.

If a veto detector were to cover the entire surface of the laboratory walls, approximately $70 \%$ first-time $(\mathrm{F})$ neutrons above $1 \mathrm{MeV}$ would register a coincident muon signal. All neutrons arrive within $\simeq 0.1 \mu$ s of the primary muon if no reflections are considered. Of the remaining (muon-unvetoed) neutron events, a further one-half contain significant $(>1 \mathrm{MeV}$ ) electromagnetic energy in the form of gamma rays crossing the boundary which can help veto the neutron. In total, only $\simeq 12 \%$ of neutron events would lack a coincident veto signal, and may therefore be difficult (or impossible) to detect. When reflected neutrons are included in this analysis $(\mathrm{F}+\mathrm{R})$ the latter fraction decreases to $8 \%$, as some neutrons thermalise enough to undergo radiative capture, making their detection easier. In this, more realistic case, neutrons above $1 \mathrm{MeV}$ enter the cavern up to $\simeq 1 \mu$ s after the primary muon, whereas lower energy neutrons can take as long as $1 \mathrm{~ms}$. 


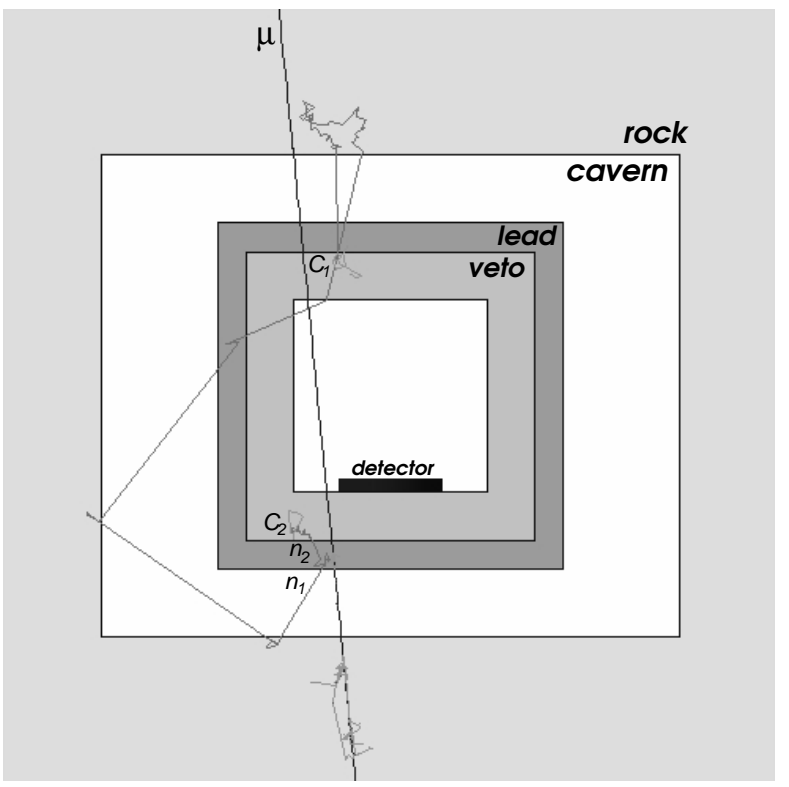

Fig. 9. Monte Carlo geometry model for a $250 \mathrm{~kg}$ liquid xenon module shielded by $50 \mathrm{~cm}$ of hydrocarbon scintillator $\left(40 \mathrm{~g} / \mathrm{cm}^{2}\right)$ and $30 \mathrm{~cm}$ of lead $\left(340 \mathrm{~g} / \mathrm{cm}^{2}\right)$. The figure also shows a GEANT4 event in which a $280 \mathrm{GeV}$ muon produces two neutrons $\left(n_{1}, n_{2}\right)$ in the lead shielding which are captured in the hydrocarbon veto $\left(C_{1}, C_{2}\right)$.

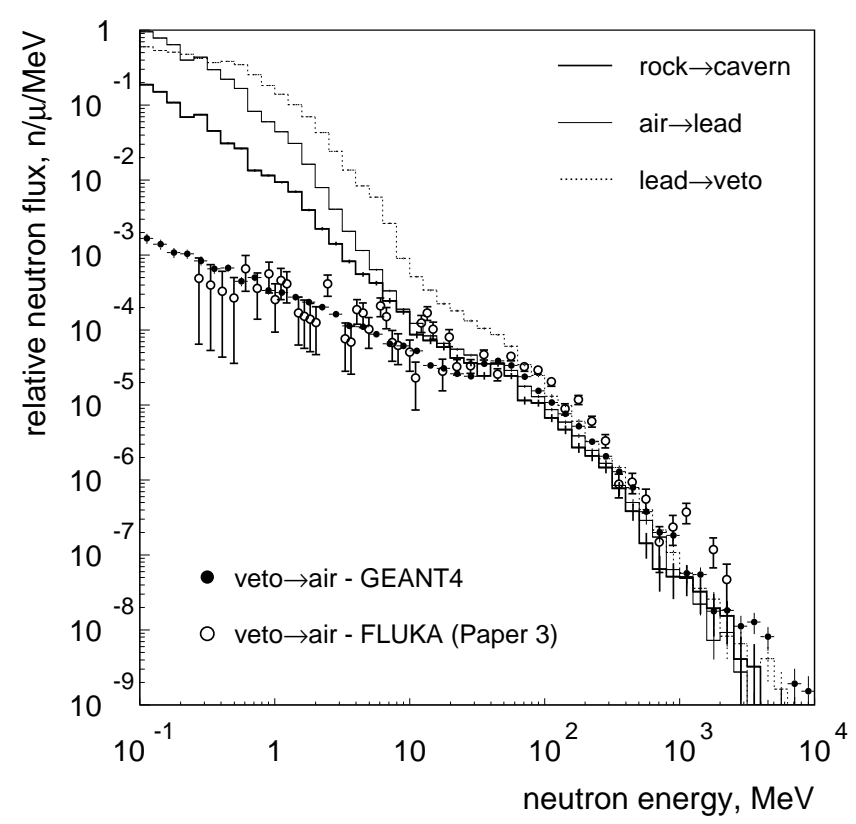

Fig. 10. Energy spectra of muon-induced neutron fluxes across several boundaries. The thick line represents the flux at the rock face for an empty cavern (curve labelled 'GEANT4 - F+R' in Fig. 8). The spectrum of neutrons exiting the hydrocarbon veto obtained with FLUKA (Paper 3) is also shown. 


\subsection{Shielding efficiency}

In the next simulation a shielded detector module is added to the empty cavern, as shown schematically in Fig. 9. A $250 \mathrm{~kg}$ liquid xenon target, contained in a copper vessel $2 \mathrm{~cm}$ thick, is surrounded by $50 \mathrm{~cm}$ of $\mathrm{C}_{10} \mathrm{H}_{20}$ scintillator to form an active veto (inner dimensions $2 \mathrm{~m} \times 2 \mathrm{~m} \times 2 \mathrm{~m}$ ) followed by $30 \mathrm{~cm}$ of lead shielding. The neutron spectra across various boundaries in this set-up are shown in Fig. 10. The figure confirms that most neutrons are produced in the lead shielding - either directly created by muons, as shown in Fig. 9, or by high-energy gammas and hadrons coming from the cavern walls or generated in the shielding itself. Although the hydrocarbon is very efficient in attenuating the neutron flux (by moderation and absorption), this lead/hydrocarbon combination provides effective shielding only for neutrons below $10 \mathrm{MeV}$. The FLUKA results presented in Paper 3 show similar trends. It should be noted that in that work the neutron spectrum at a particular boundary was calculated before a new layer is added to the geometry, e.g. the effect of the lead shielding is calculated before an inner hydrocarbon layer is introduced (and so the difference to the one shown here is greater than a factor of $\simeq 2$ for the total production rate in Fig. 5). Similarly, the neutron flux exiting the hydrocarbonbased veto was calculated before the detector is added to the model. Given the small size of the detector itself compared to the inner surface of the veto, the comparison shown in Fig. 10 is appropriate. The neutron spectra emerging across the veto calculated by the two MCs are in good agreement, especially for neutron energies below a few tens of $\mathrm{MeV}$.

\subsection{Neutron detection in veto and xenon target}

The prompt scintillation signal is the detection mechanism now considered for the liquid xenon target. To account for quenching of the scintillation yield for nuclear recoils relative to electron-recoil interactions, a quenching factor of 0.2 is applied to the energy deposited in neutron elastic scattering events [24,25]. We assign a detection threshold of $2 \mathrm{keV}$ electron-equivalent (e.e.) or 'visible' energy to the target, which is a realistic scenario for xenon WIMP detectors. In the preceding analysis the hydrocarbon scintillator had the role of passive shielding. We now consider it as an active veto, which is a much more powerful way to reduce the neutron background in the xenon target. A threshold of $100 \mathrm{keV}$ e.e. is assumed for detection of proton recoils (and other particles) in a typical veto using this type of organic scintillator $(500-700 \mathrm{keV}$ proton recoil

energy [26]). Such an idealised veto system, with full angular coverage, can reach high rejection efficiency $[27,28]$ of neutrons produced in detector components, by detecting proton recoils and gamma rays from radiative neutron capture - two such capture events are illustrated in Fig. 9. 


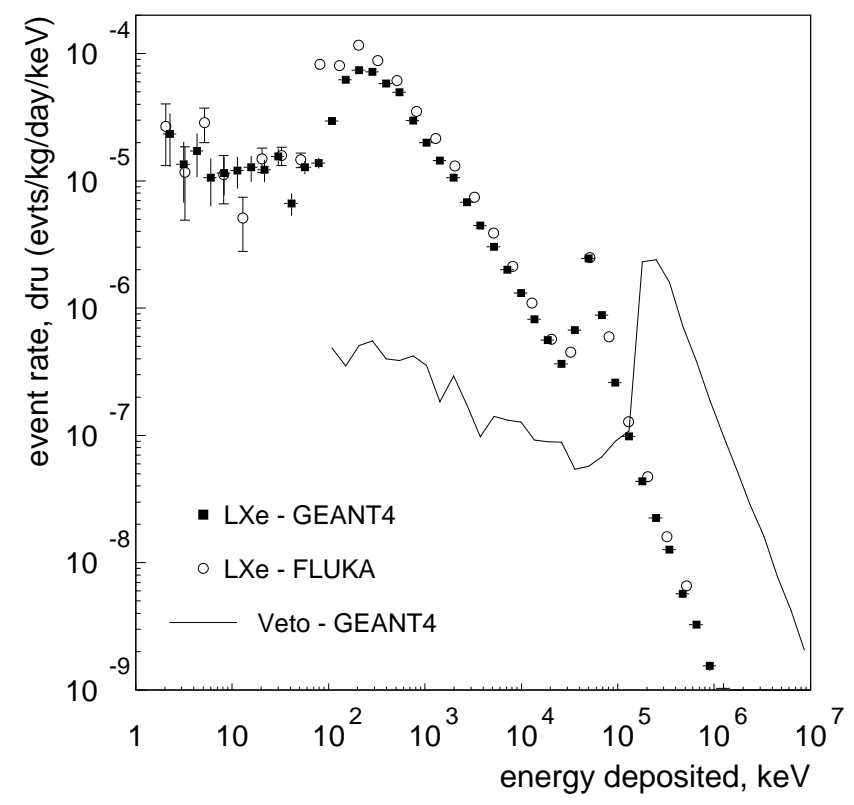

Fig. 11. Differential spectra of the total energy deposited in the liquid xenon (LXe) target as predicted by GEANT4 and FLUKA and in the veto scintillator according to GEANT4 (the latter is scaled down by a factor of $5 \times 10^{4}$ ).

The total energy deposition spectra in the xenon target according to both MC codes are plotted in Fig. 11. This is mainly 'electromagnetic' energy: nuclear recoils have a noticeable contribution only at the lowest energies. The simulations agree within statistical errors in the low-energy region below $100 \mathrm{keV}$. The discrepancy observed near $100 \mathrm{keV}$ may be due to isotope-production followed by radioactive decay with gamma emission, which was modelled in the FLUKA simulation but not considered in this GEANT4 work. FLUKA predicts more gammas than GEANT4 between $100 \mathrm{keV}$ and a few tens of MeV - which is not surprising considering that it also produces more neutrons but the general agreement is still reasonable. At higher energies, where the passage of primary muons causes the peak seen in the figure, the two spectra nearly coincide. The spectrum of the total energy deposited in the veto is also shown (uncorrected by the quenching factor in the case of proton recoils).

To obtain a statistically significant dataset of nuclear recoil events, an exposure time of nearly 3 years was simulated with GEANT4 ( $\simeq 20 \times 10^{6}$ muons). FLUKA simulations were performed with the new release FLUKA-2003 giving the statistics of about 4 years of live time. The nuclear recoil spectrum in the xenon target is shown in Fig. 12. The spectra include both 'pure' and 'mixed' recoil events, the latter term representing events involving both electromagnetic energy and nuclear recoils. The total number of recoils with any energy predicted by GEANT4 for the $250 \mathrm{~kg}$ target is $217 \pm 9$ per year, of which $10.3 \pm 1.9$ are not accompanied by additional energy deposits. In the visible energy range of $2-10 \mathrm{keV}$, where most WIMP signals should occur, 


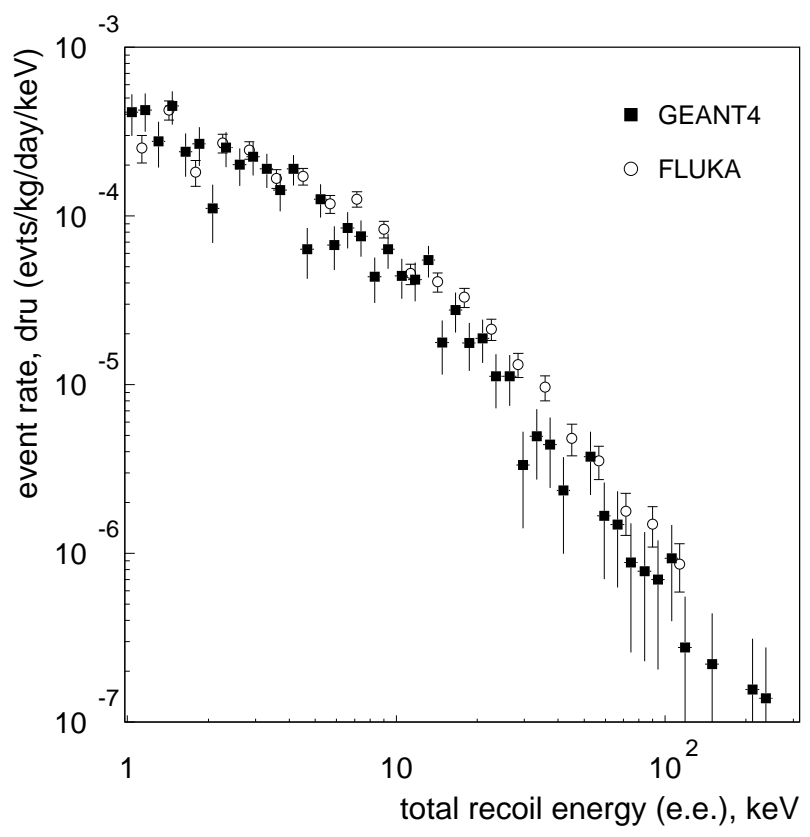

Fig. 12. Nuclear-recoil energy spectrum in the liquid xenon detector as a function of the visible energy deposited by all nuclear recoils in each event. The spectra include 'mixed' events involving electromagnetic energy deposits, not just 'pure' nuclear recoils, but only the energy left by nuclear recoils was counted.

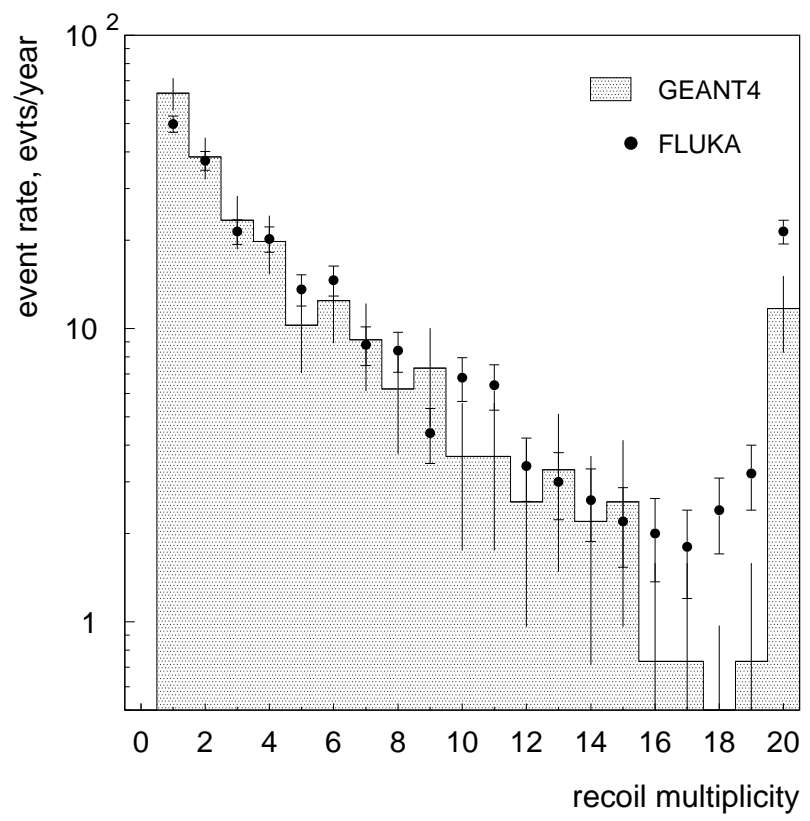

Fig. 13. Distribution of the nuclear-recoil multiplicity in the $250 \mathrm{~kg}$ xenon target, for all events containing neutron elastic scatters. The last data-point includes multiplicities of 20 and above.

$3.3 \pm 1.1$ recoils are expected per year. A similar figure of $3.9 \pm 1.0$ is predicted by FLUKA. The results from the two codes are compared in Table 1. 
Table 1

Muon-induced neutron background (nuclear recoil (NR) events per year) in $250 \mathrm{~kg}$ xenon target for several detection thresholds

\begin{tabular}{l|c|cc}
\hline \hline Event type & $E_{e e}, \mathrm{keV}$ & FLUKA & GEANT4 \\
\hline All NR events & $>0$ & $234 \pm 8$ & $217 \pm 9$ \\
Pure NR events & $>0$ & $13.6 \pm 1.9$ & $10.3 \pm 1.9$ \\
& $>2$ & $7.3 \pm 1.4$ & $4.0 \pm 1.2$ \\
& $2-10$ & $3.9 \pm 1.0$ & $3.3 \pm 1.1$ \\
Pure single NR events & $>0$ & $6.6 \pm 1.3$ & $6.6 \pm 1.6$ \\
& $2-10$ & $2.1 \pm 0.7$ & $2.6 \pm 1.0$ \\
Anti-coincidence with veto & $>0$ & 0 & 0 \\
\hline
\end{tabular}

Note that the numbers presented in Table 1 for FLUKA agree with the previous simulations (Paper 3 ) for the total recoil rate, but are a factor of 2 smaller for 'pure' nuclear recoil events. This is due to the lower energy cuts for gammas and electrons used in the present simulations $(50 \mathrm{keV}$ for xenon and its vessel, $100 \mathrm{keV}$ for hydrocarbon and lead and $1 \mathrm{MeV}$ for rock). This makes the probability of coincidences between nuclear recoils and gammas/electrons in xenon higher, and reduces the rate of 'pure' nuclear recoils.

A detector with good spatial resolution can resolve the locations of multiple neutron elastic interactions, which occur on average several $\mathrm{cm}$ apart. These are not regarded as irreducible background since they should not be caused by WIMPs. The recoil multiplicity in the target (i.e. the distribution of the number of recoils per MC event) is shown in Fig. 13 (including all recoil events, of all energies). GEANT4 predicts that $28 \%$ of these are single recoils. Considering only those with visible energies greater than $2 \mathrm{keV}$ - i.e. which have a realistic chance of being detected - the fraction of single scatters decreases to $14 \%$. Finally, the simulation registered $2.6 \pm 1.0$ single recoils per year in the energy range $2-10 \mathrm{keV}$ e.e., whereas FLUKA indicates $2.1 \pm 0.7$ recoils per year. In both MCs all recoil events were vetoed with a signal greater than $100 \mathrm{keV}$, i.e. no recoils were observed in the target in anti-coincidence with the veto.

In spite of predicting different neutron yields, the results summarised in Table 1 suggest that FLUKA and GEANT4 arrive at a similar total number of neutron events, as well as similar rates in the range $2-10 \mathrm{keV}$ e.e. This is explained by the neutron spectra at the rock face shown in Fig. 10: although the total yields from FLUKA can be a factor of 2 higher in materials such as salt and lead, the actual integral flux above $1 \mathrm{MeV}$, capable of producing detectable nuclear recoils, differs by about $20-30 \%$. 


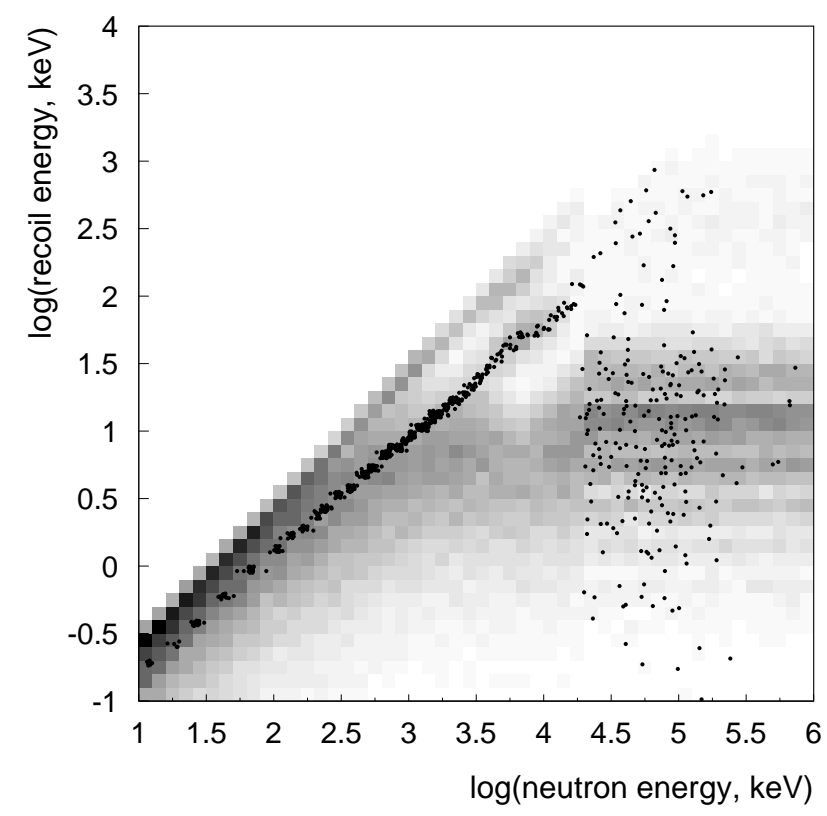

Fig. 14. Kinetic energy of xenon recoils as a function of neutron energy in the FLUKA simulation (filled markers) and for a log-uniform neutron distribution with GEANT4 (grey-scale). In both MC codes, neutron elastic scattering is treated by different models below and above $\simeq 20 \mathrm{MeV}$.

Although the nuclear recoil spectrum is generally harder in FLUKA than in GEANT4, the number of events at $2-10 \mathrm{keV}$ does not differ much in the two models. The difference between these spectra is mainly explained by the different treatment of neutron elastic scattering in the two codes. The databased models describing elastic interactions below $20 \mathrm{MeV}$ are more accurate in GEANT4 than in FLUKA. This is illustrated in Fig. 14, which plots the nuclear recoil energies in the xenon target obtained with FLUKA against the corresponding neutron energy. A new dataset was generated with GEANT4 for a log-uniform neutron energy distribution, also shown in the figure. Both MCs exhibit a discontinuity at $\simeq 20 \mathrm{MeV}$, where parameterised models take over from the low-energy ones. The most striking difference is that FLUKA generates mono-energetic recoils (based on Kerma factors) for a fixed neutron energy in the low energy region, whereas GEANT4 is much more accurate, reproducing the full recoil spectrum.

\section{Conclusions}

We have investigated how GEANT4 and FLUKA model muon-induced neutrons in bulk matter in an attempt to validate these Monte Carlo codes for the simulation of the neutron backgrounds expected in underground laboratories. The total yields, neutron energy spectrum and lateral distribution from the 
muon track in hydrocarbon material obtained with both codes are consistent with the available measurements, with GEANT4 predicting fewer neutrons than FLUKA above $100 \mathrm{GeV}$ muon energy by a small factor $(<30 \%)$. In most other materials studied, GEANT4 also generates lower neutron fluxes for muon energies in the hundreds of $\mathrm{GeV}$. At $280 \mathrm{GeV}$, the typical average muon energy at several underground laboratories, the total neutron yield can be a factor of 2 lower for some materials. These include salt and lead, which are important as rock and shielding media. In general, GEANT4 shows enhanced neutron photoproduction in electromagnetic cascades in light materials, although this tendency is less marked relative to the recent FLUKA release. For heavy elements, FLUKA produces more neutrons than GEANT4 in both electromagnetic and hadronic cascades initiated by muons. A new theory-driven model for the hadronic vertex of the muon-nucleus interaction will appear in a future GEANT4 release [16], which may increase the hadronic yields. Experimental yields obtained for $\mathrm{Pb}[18,19]$ appear to be mutually inconsistent, but may suggest that both simulations underestimate neutron production in heavy targets. Direct production in muon spallation reactions is similar in both codes, although the simulated rates for fast neutrons $(>10 \mathrm{MeV})$ disagree with experimental data obtained in the CERN NA55 experiment. Such marked disagreement is not observed with measurements by the E665 Collaboration for neutrons under $10 \mathrm{MeV}$.

The two packages were used to model the muon-induced background in an idealised WIMP detector containing $250 \mathrm{~kg}$ of liquid xenon as an active medium. The simulated total fluxes of fast neutrons $(>1 \mathrm{MeV})$ entering the underground cavern were found to agree within $20 \%$. Similar agreement was obtained for the neutron flux emerging after $30 \mathrm{~cm}$ of lead and $50 \mathrm{~cm}$ of hydrocarbon shielding. The nuclear recoil spectrum observed in the xenon target is slightly harder in the FLUKA simulation, which also predicts a small excess of 'pure' recoil events. However, both simulations indicate a similar number of recoils with visible energy of $2-10 \mathrm{keV}$. A single-recoil rate of the order of 2 events/year is predicted by both models, which is probably not far below the value expected from an 'ideal' Monte Carlo code. We do not expect a large enhancement in the neutron flux after all possible improvements to the codes have been implemented.

\section{Acknowledgments}

This work has been undertaken within the framework of the UK Dark Matter Collaboration (University of Edinburgh, Imperial College London, Rutherford Appleton Laboratory and University of Sheffield) and contributes to the collaboration-wide simulation efforts. The work is supported by the UK Particle Physics and Astronomy Research Council (PPARC). The authors wish 
to thanks H. P. Wellisch (CERN) for his invaluable assistance and for his comments to this manuscript. Thanks are due to M. Robinson (University of Sheffield) and L. Mendes (Imperial College) for their help with computational facilities. We also acknowledge the funding from the EU FP6 project ILIAS.

\section{References}

[1] A.Fassò, A.Ferrari and P.R.Sala, in: Proceedings of the MonteCarlo 2000 Conference (Lisbon, October 23-26, 2000), Ed. A.Kling, F.Barao, M.Nakagawa, L.Távora, P.Vaz (Springer-Verlag, Berlin, 2001), p. 159; A.Fassò, A.Ferrari, J.Ranft and P.R.Sala, ibid. p. 995.

[2] Geant4 Collaboration, Nucl. Instrum. Meth. A 506 (2003) 250;

[3] V.A.Kudryavtsev, N.J.C.Spooner \& J.E.McMillan, Nucl. Instrum. Meth. A 505 (2003) 688. (Paper 1)

[4] Y.-F.Wang et al., Phys. Rev. D 64 (2001) 013012. (Paper 2)

[5] M.J.Carson et al., Astroparticle Phys. 21(6) (2004) 667. (Paper 3)

[6] GEANT4 Physics Reference Manual, available from http://cern.ch/geant4.

[7] P.Antonioli, C.Ghetti, E.V.Korolkova, V.A.Kudryavtsev and G.Sartorelli, Astroparticle Phys. 7 (1997) 357.

[8] H.Araújo \& V.A.Kudryavtsev, talk at the 5th Int. Workshop on the Identification of Dark Matter (Edinburgh, UK, 6-10 September 2004) IDM2004, available at http://www.shef.ac.uk/physics/idm2004.html.

[9] M.Aglietta et al., Proc. 26th Int. Conf. Cosmic Rays, Salt Lake City, Vol. 2 (1999) 44; hep-ex/9905047.

[10] V.Chazal et al., Nucl. Instrum. Meth. A 490 (2002) 334.

[11] V.V.Borog \& A.A.Petrukhin, Proc. 14th Int. Conf. Cosmic Rays, Munich, Vol. $6(1975) 1949$.

[12] L.B.Bezrukov \& E.V.Bugaev, Sov. J. Nucl. Phys. 33 (1981) 635.

[13] D.O.Caldwell et al., Phys. Rev. Lett. 42, (1979) 553.

[14] Muon Photonuclear Interaction, in: GEANT4 Physics Reference Manual.

[15] H.Menghetti (LVD Collaboration), talk at the 5th Int. Workshop on the Identification of Dark Matter (Edinburgh, UK, 6-10 September 2004) IDM2004, available at http://www.shef.ac.uk/physics/idm2004.html.

[16] H.P.Wellisch (CERN), private communication.

[17] H.Wulandari et al., hep-ex/0401032. 
[18] G.V.Gorshkov et al., Sov. J. Nucl. Phys. 7 (1968) 470; ibid. 12 (1971) 187; ibid. 13 (1974) 450; ibid. 18 (1974) 57.

[19] L.Bergamasco, S.Costa \& P.Picchi, Il Nuovo Cimento 13A (1973) 403.

[20] M.R.Adams et al., Phys. Rev. Lett 74(26) (1995) 5198; also erratum, in: Phys. Rev. Lett 80(9) (1998) 2020;

[21] G.Gerbier, talk at the 5th Int. Workshop on the Identification of Dark Matter (Edinburgh, UK, 6-10 September 2004) - IDM2004, available at http://www.shef.ac.uk/physics/idm2004.html.

[22] V.A.Kudryavtsev et al., in: Proc. 4th Int. Workshop on the Identification of Dark Matter (York, UK, 2-6 Sep 2002), Eds. N.J.C.Spooner \& V.Kudryavtsev (World Scientific, Singapore, 2003), p.477.

[23] M.Robinson et al., Nucl. Instrum. Meth. A 511 (2003) 347.

[24] D.Akimov et al., Physics Letters B 524 (2002) 245.

[25] F.Arneodo et al., Nucl. Instrum. Meth. A 449 (2000) 147.

[26] M.Anghinolfi et al., Nucl. Instrum. Meth. A 165 (1979) 217.

[27] P.F.Smith et al., Astroparticle Phys., in press.

[28] M.Carson et al., submitted to Nucl. Instrum. Meth. (2004). 